\title{
Routing System With Diversion in Wireless Ad Hoc Security
}

\author{
R. Divya Vani1)
}

\begin{abstract}
Author considered the issue of routing packets across a multi-hop network comprising of numerous wellsprings of movement and remote connections while guaranteeing limited expected postponement. Every bundle transmission can be caught by an irregular subset of collector hubs among which the following transfer is chosen deftly. The primary test in the plan of least postponement steering strategies is adjusting the exchange off between directing the parcels along the briefest ways to the goal and circulating the activity as per the greatest backpressure. Joining critical parts of most limited way and backpressure steering, this paper gives a deliberate advancement of a disseminated pioneering directing arrangement with clog differences (D-ORCD). D-ORCD utilizes a measure of depleting time to sharply distinguish and course parcels along the ways with a normal low general clog. D-ORCD with single goal is demonstrated to guarantee a limited expected deferral for all systems and under any allowable movement, inasmuch as the rate of computations is adequately quick in respect to activity insights. Besides, this paper proposes a commonsense usage of D-ORCD which experimentally advances basic calculation parameters and their consequences for postponement and also convention overhead. Reasonable QualNet reenactments for 802.11-based systems show a critical change in the average delay over comparable solutions in the literature.
\end{abstract}

Keywords: congestion measure, implementation, Lyapunov analysis, opportunistic routing, queuing stability, wireless ad hoc networks.

\section{Introduction}

OPPORTUNISTIC routing for multi-hop wireless ad hoc networks has for quite some time been proposed to beat the deficiencies of conventional routing[1-5]. Artful directing mitigates the effect of poor remote connections by abusing the communicate way of remote transmissions and the way differing qualities. All the more exactly, the pioneering directing choices are made in an online way by picking the following transfer in light of the real transmission results and in addition a rank requesting of neighboring hubs. The creators in[4] gave a Markov choice theoretic definition for pioneering steering and a bound together system for some adaptations of shrewd directing [1-3], with the varieties because of the creators' selections of expenses.

Received(October 29, 2015), Review Result(1st: October 30, 2015, 2nd: November 19, 2015), Accepted(December 10, 2015)

${ }^{1}$ (Corresponding Author) Vetri Vikas Educational Institutions, Mallur (Via), Rasipuram Taluk, Annamalaipatti, Tamil Nadu, India email: Divyavani002@gmail.com 
Specifically, it is demonstrated that for any bundle, the ideal steering choice, in the feeling of least cost or bounce tally, is to choose the following hand-off hub in view of a list. This file is equivalent to the normal cost or jump check of handing-off the bundle along the slightest expensive or the most brief practical way to the goal. At the point when various floods of parcels are to cross the system, be that as it may, it may be alluring to course a few bundles along longer or all the more exorbitant ways, if these ways inevitably prompt to connections that are less congested. All the more unequivocally, as noted in [6-7], the pioneering steering plans in [1-5] can conceivably bring about extreme blockage and unbounded deferral (see the cases given in [6]). Conversely, it is realized that a shrewd variation of backpressure [8], differences backpressure steering (DIVBAR) [7] guarantees limited expected aggregate excess for all stabilizable entry rates. To guarantee throughput optimality (limited expected aggregate excess for all stabilizable landing rates), backpressure-based calculations [7][8] accomplish something altogether different from [1-5]: as opposed to utilizing any metric of closeness (or cost) to the goal, they pick the collector with the biggest positive differential accumulation (steering duty is held by the transmitter if no such beneficiary exists). This very property of disregarding the cost to the goal, be that as it may, turns into the most despicable aspect of this approach, prompting to poor defer execution in low to direct movement (see [6]). Other existing provably throughput optimal routing policies [9-12] disseminate the movement locally in a way like DIVBAR and subsequently, result in large delay.

\section{System architecture}

\subsection{Existing System}

The sharp steering plans can conceivably bring about extreme blockage and unbounded deferral. Conversely, it is realized that a deft variation of backpressure, assorted qualities backpressure steering (DIVBAR) guarantees limited expected aggregate excess for all stabilizable entry rates. To guarantee throughput optimality (limited expected aggregate excess for all stabilizable landing rates), backpressure-based calculations accomplish something altogether different: instead of utilizing any metric of closeness (or cost) to the goal, they pick the recipient with the biggest positive differential accumulation (directing duty is held by the transmitter if no such beneficiary exists).

E-DIVBAR is proposed: while picking the following hand-off among the arrangement of 
potential forwarders, E-DIVBAR considers the sum of the differential backlog and the expected hop-count to the destination (otherwise called ETX).

\subsection{Disadvantages of Existing System}

The existing property of overlooking the cost to the destination, be that as it may, turns into the most despicable aspect of this approach, prompting to poor defer execution in low to moderate traffic.

Other existing provably throughput ideal steering approaches appropriate the activity locally in a way like DIVBAR and consequently, result in vast postponement.

\subsection{Proposed System}

The primary commitment of this paper is to give a distributed opportunistic routing policy with congestion diversity (D-ORCD) under which, rather than a basic expansion utilized as a part of E-DIVBAR, the clog data is incorporated with the circulated most limited way calculations. A thorough examination of the execution of D-ORCD is given in two directions:

Author gave detailed simulation investigation of defer execution of D-ORCD. We additionally handle a portion of the framework level issues observed in realistic settings via detailed simulations.

Notwithstanding the simulation studies, we demonstrate that D-ORCD is throughput ideal when there is a solitary goal (single ware) and the system works in stationary administration. While portraying postpone execution is frequently not logically tractable, numerous variations of backpressure algorithm are known to accomplish throughput optimality.

\subsection{Advantages of Proposed System:}

Author demonstrates that D-ORCD shows preferred delay performance than state-of-the-art routing strategies with comparable unpredictability, specifically, ExOR, DIVBAR, and E-DIVBAR. We likewise demonstrate that the relative execution change over existing arrangements, all in all, relies on upon the system topology yet is regularly huge by and by, where flawlessly symmetric system sending and activity conditions are unprecedented.

Author demonstrated that a similar analytic assurance can be gotten with respect to the throughput optimality of D-ORCD. Specifically, we demonstrate the throughput optimality of 
D-ORCD by taking a gander at the meeting of D-ORCD to a unified form of the calculation. The optimality of the centralized solution is established via a class of Lyapunov functions proposed.

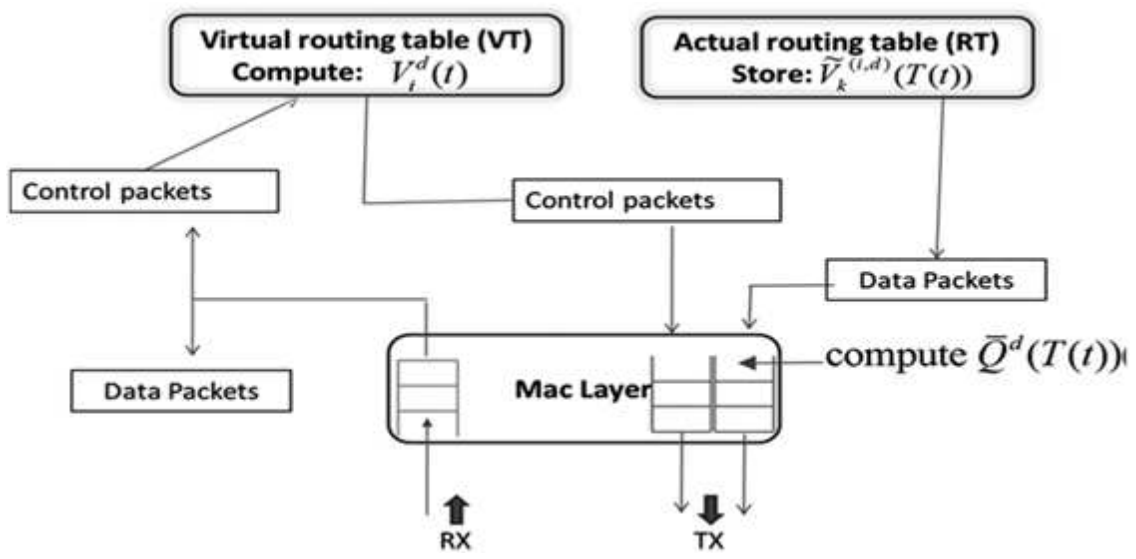

[Fig. 1] System Architecture

\section{Implementation Modules Description}

\subsection{System Formation}

In this module, initially author tends to develop the System Formation ideas. Author tends to think about a network of $\mathrm{D}$ nodes. And author tends to characterize the behavior of the wireless channel employing a probabilistic transmission model. Node is alleged to be neighbor of node, if there's a positive chance FTO that a transmission at node $\mathrm{i}$ is received at node. The set of all nodes within the network that square measure approachable by node is remarked as neighborhood of node.

D-ORCD depends on a routing table at every node to see consequent best hop. The routing table at node consists of an inventory of neighbors and a structure consisting of calculable congestion live for all neighbors in related to completely different destinations.

The routing table acts as a storage and call element at the routing layer. The routing table is updated employing a "virtual routing table" at the tip of each "computation cycle": Associate in Nursing interval of units of your time.

To update virtual routing table, throughout the progression of the computation cycle the nodes exchange and calculate the temporary congestion measures. 


\subsection{Congestion live}

In this module, author tends to develop the planned system by this the system will able to establish the Congestion happened. The congestion live values square measure code and outlined within the module.

The congestion measure associated with node for a destination at time is the aggregate sum of the local draining time at node and the draining time from its next hop to the destination. D-ORCD computes the expected congestion measure "down the stream".

The implementation of $\mathrm{D}-\mathrm{ORCD}$, analogous to any opportunistic routing scheme, involves the selection of a relay node among the candidate set of nodes that have received and acknowledged a packet successfully. One of the major challenges in the implementation of an opportunistic routing algorithm, in general, and D-ORCD in particular, is the design of an 802.11 compatible acknowledgement mechanism at the MAC layer.

\subsection{Link Quality Estimation Protocol}

In this module, author develops the Link Quality Estimation Protocol for the proposed system model. D-ORCD computations given by (1) utilize link success probabilities pij for each pair of nodes i,j. We now describe a method to determine the probability of successfully receiving a data packet for each pair of nodes.

Our method consists of two components: active probing and passive probing.

In the active probing, dedicated probe packets are broadcasted periodically to estimate link success probabilities.

In passive probing, the overhearing capability of the wireless medium are utilized. The nodes are configured to promiscuous mode, hence enabling them to hear the packets from neighbors. In passive probing, the MAC layer keeps track of the number of packets received from the neighbors including the retransmissions.

Finally, a weighted average is used to combine the active and passive estimates to determine the link success probabilities. Passive probing does not introduce any additional overhead cost but can be slow, while active probing rate is set independently of the data rate but introduces costly overhead. 


\subsection{Opportunistic Routing With Partial Diversity}

In the module, the opportunistic Routing part is implemented and developed in the proposed system model. The three-way handshake procedure achieves opportunism and receiver diversity gain at the cost of an increased feedback overhead. In particular, it is easy to see that this overhead cost, i.e., the total number of ACKs sent per data packet transmission, increases linearly with the size of the set of potential forwarders. Thus, we consider a modification of D-ORCD in the form of opportunistically routing with partial diversity (PD-ORCD).

This class of routing policies is parametrized by a parameter denoting the maximum number of forwarder nodes: the maximum number of nodes allowed to send acknowledgment per data packet transmission is constrained to be no more than. Such a constraint will sacrifice the diversity gain, and hence the performance of any opportunistic routing policy, in favor of lowering overhead cost.

In order to implement opportunistic routing policies with partial diversity, before the transmission stage occurs, we find the set of "best neighbors" for each node.

\section{Conclusion}

In this paper, author gave a distributed opportunistic routing policy with congestion diversity (D-ORCD) by consolidating the critical parts of most limited way directing with those of backpressure routing. Under this arrangement parcels are directed by a rank requesting of the hubs in light of a clog measure. Besides, author proposed a practical distributed and asynchronous 802.11 good usage of D-ORCD, whose execution was explored by means that of a precise arrangement of QualNet reproductions for pragmatic and sensible systems. Reenactments incontestable that D-ORCD faithfully beats existing leading calculations. author tended to likewise gave hypothetic turnout optimality verification of D-ORCD. In D-ORCD, author does not show the resistance from the hubs within the system, nonetheless rather leave that issue to a longtime mack operation. The speculation to the systems with between channel resistance seem to require once squarely from [7] where, the value of this speculation is looked as if it would be the centralization of the routing/scheduling internationally over the system or a gradual part execution loss of the distributed variations [7][10]. In future, we tend to square measure keen on summary D-ORCD for joint steering and booking enhancements too considering the framework level ramifications. Fusing turnout ideal CSMA primarily based 
mack hardware with blockage aware leading is to boot encouraging region of analysis.

The define of D-ORCD needs data of channel statistics. coming up with clog management routing algorithms to reduce expected delay while not the topology and therefore the channel statistics information is a neighborhood of future analysis.

\section{References}

[1] P. Larsson, Selection diversity forwarding in a multihop packet radio network with fading channel and capture, ACM SIGMOBILE Mobile Comput. Commun. Rev., (2001), Vol.5, No.4, pp.47-54.

[2] M. Zorzi and R. R. Rao, Geographic random forwarding (GeRaF) for Ad Hoc and sensor networks: Multihop performance, IEEE Trans. Mobile Comput., (2003), Vol.2, No.4.

[3] S. Biswas and R. Morris, ExOR: Opportunistic multi-hop routing for wireless networks, ACM SIGCOMMComput. Commun. Rev., (2005), Vol.35, pp.33 - 44, Oct.

[4] C. Lott and D. Teneketzis, Stochastic routing in ad hoc networks, IEEE Trans. Autom. Contr., (2006), Vol.51, pp.52-72.

[5] S. Jain and S. R. Das, Exploiting path diversity in the link layer in wireless ad hoc networks, in Proc. WoWMoM, (2005), pp.22-30.

[6] P. Gupta and T. Javidi, Towards throughput and delay optimal routing for wireless ad hoc networks, in Proc. Asilomar Conf., (2007), pp.249-254.

[7] M. J. Neely and R. Urgaonkar, Optimal backpressure routing for wireless networks with multi-receiver diversity, Ad Hoc Networks, (2009), Vol.7, No.5, pp.862-881.

[8] L. Tassiulas and A. Ephremides, Stability properties of constrained queueing systems and scheduling policies for maximum throughput in multihop radio networks, IEEE Trans. Autom. Contr., (1992), Vol.37, No.12, pp.1936-1949.

[9] S. Sarkar and S. Ray, Arbitrary throughput versus complexity tradeoffs in wireless networks using graph partitioning, IEEE Trans. Autom. Contr., (2008), Vol.53, No.10, pp.2307-2323.

[10] Y. Xi and E. M. Yeh, Throughput optimal distributed control of stochastic wireless networks, in Proc. 4th Int. Symp. Modeling and Optimization in Mobile, Ad Hoc, and Wireless Networks (WiOpt), (2006), pp.1-10.

[11] B. Smith and B. Hassibi, Wireless erasure networks with feedback, arXiv: 0804.4298v1, (2008).

[12] Y. Yi and S. Shakkottai, Hop-by-hop congestion control over a wireless multi-hop network, IEEE/ACM Trans. Netw., (2007), Vol.15, No.1, pp.133 - 144. 